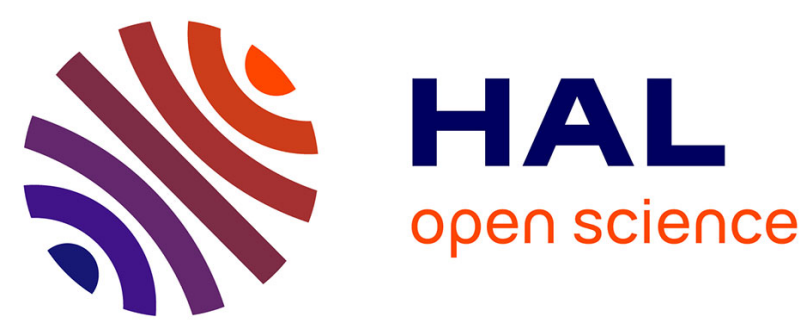

\title{
A New Framework for Efficient Shared Segment Protection Scheme for WDM Networks
}

Brigitte Jaumard, Nazmun Nahar Bhuiyan, Samir Sebbah, Florian Huc, David Coudert

\section{- To cite this version:}

Brigitte Jaumard, Nazmun Nahar Bhuiyan, Samir Sebbah, Florian Huc, David Coudert. A New Framework for Efficient Shared Segment Protection Scheme for WDM Networks. 11th International Conference on High Performance Switching and Routing (HPSR), Jun 2010, Richardson, Texas, USA, Canada. pp.8, 10.1109/HPSR.2010.5580274 . inria-00482119

\section{HAL Id: inria-00482119 https://hal.inria.fr/inria-00482119}

Submitted on 2 Nov 2010

HAL is a multi-disciplinary open access archive for the deposit and dissemination of scientific research documents, whether they are published or not. The documents may come from teaching and research institutions in France or abroad, or from public or private research centers.
L'archive ouverte pluridisciplinaire HAL, est destinée au dépôt et à la diffusion de documents scientifiques de niveau recherche, publiés ou non, émanant des établissements d'enseignement et de recherche français ou étrangers, des laboratoires publics ou privés. 


\title{
A New Framework for Efficient Shared Segment Protection Scheme for WDM Networks
}

\author{
Brigitte Jaumard*, Nazmun Nahar Bhuiyan ${ }^{\dagger}$, Samir Sebbah ${ }^{\ddagger}$, Florian Huc ${ }^{\S}$, David Coudert $₫$ \\ * GERAD, CIRRELT, and CIISE, Concordia University, Montréal, Canada \\ $\dagger$ CSE, Concordia University, Montréal, Canada \\ $\ddagger$ ECE, Concordia University, Montréal, Canada \\ $\S$ TCS-sensor lab, Computer Science Department, Université of Genève, Switzerland \\ I Mascotte project, I3S(CNRS/UNSA)/INRIA, Sophia-Antipolis, France
}

\begin{abstract}
This work introduces a new shared segment protection scheme that ensures both node and link protection in an efficient manner in terms of cost and bandwidth, while taking full advantage of the optical hop endpoints of the primary logical hops (induced by the routing) without adding extra ones for protection. As opposed to the link or path protection schemes, the segment protection scheme has been less studied although it offers an interesting compromise between those two protection schemes, attempting to encompass all their advantages. We investigate two different Shared Segment Protection (SSP) schemes: Basic Shared Segment Protection (BSSP) and Shared Segment Protection with segment Overlap (SSPO), and propose design of $100 \%$ single segment protections. In SSPO, we study the extra protection capabilities, node failure and dual link failure survivability, offered by the single $100 \%$ segment protection.

For both BSSP and SSPO schemes, we propose two novel efficient ILP formulations, based on a column generation mathematical modeling. While (SSPO) offers the advantage over (BSSP) to ensure both node and link protection, it is not necessarily much more costly. Indeed, depending on the network topology and the traffic instances, it can be shown that none of the two SSP schemes dominates the other one. Therefore, the SSPO protection scheme should be favored as it offers more protection, i.e., it adds the node protection to the link protection at the expense of a minor additional cost.
\end{abstract}

\section{INTRODUCTION}

Modern transport networks are based on WDM technology, where reconfigurable Optical Cross Connects (OXC) and Optical Add-Drop Multiplexers (OADM) are getting widely deployed in order to respond to the growing demand for high bandwidth services [1]. Under WDM, optical switches are equipped with optical transceivers tuned to different wavelengths and bypass capabilities in order to set up end-to-end connections - also called lightpaths - in the network. The Routing and Wavelength Assignment (RWA) problem consists thus in assigning a route in the network and a wavelength to each lightpath. This NP-hard problem has been widely studied during the last 15 years and optimal or near-optimal solutions are now available (e.g., [2]).

The Routing and Wavelength Assignment (GRWA) problem ([3], [4]) is another related problem of joint bandwidth management and routing in optical WDM networks. SONET and Optical Transport Network (ONT) are two standards that perform wavelength management by grooming finer granularity connections into a coarse wavelength channel.
With the event of WDM systems, hundreds of wavelengths are switched at optical nodes. This has opened up another problem of optical switching capabilities in the system. Two different switching architectures exist and are characterized by the switching domain of their signals. In the electrical switching architecture, switching nodes are equipped for each wavelength with an input and output port (transceiver) that switch the signal in the electrical domain. This architecture can support more functionalities, i.e., grooming, signal re-shaping. However, it is expensive as transceivers are the dominant cost factor. Another switching architecture is the transparent optical (photonic), where bypassing of signal is enabled through intermediate nodes along the lightpaths. This solution is further more scalable than the previous one, however, it is of limited reach, i.e., optical signal cannot travel long distance without undergoing regeneration.

In this current study, we propose a protection scheme that can span both switching architectures while taking full advantage of both of them in terms of grooming, regeneration, and switching scalability. Our protection approach is based on Shared Segment Protection schemes. In this context, we propose a sequential design approach where the working segments are first defined (using any given GRWA algorithm) and then the protection scheme is optimized. We therefore assume that we are given a set of working paths, where each working path is either single-hop or multi-hop, i.e., made of one or several working segments. In the present study, we limited ourselves to no more than 3 segments. It is justified by the end-to-end delay requirements, i.e., no more than two electronic/optical/electronic conversion between the source and the destination of a request. Transport blades/ports are installed at each endpoint of a working segment. This induces a natural segmentation of the lightpath that can be used as a base for the protection scheme in order to save on the network cost.

A first segment protection, called BSSP, is such that a protection is defined for each working segment, and therefore such that both working and protection segments have the same endpoints. A second segment protection, called SSPO, is such that for 3-hop working paths, we allow protection segments to encompass two working segments in order to reduce the bandwidth cost, but also and firstly to ensure node 


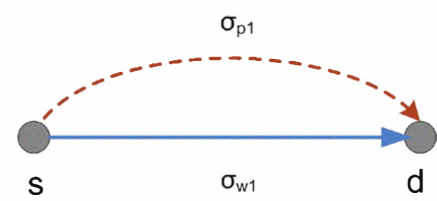

(a) Single hop request

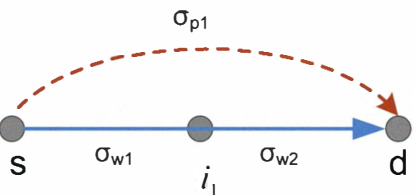

(b) Two hop request

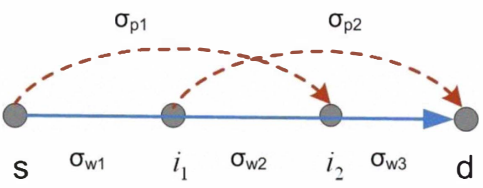

(c) Three hop request

Fig. 1. SSPO protection scheme

protection. This entails some overlapping of the protection segments. In the next section, we will compare SSPO with the SLSP protection scheme of Ho and Mouftah [5] where the overlapping is at the level of the working segments. In both BSSP and SSPO, protection paths can be shared by several node and link disjoint working segments, it is illustrated in Figure 2(b) where $\sigma_{p}$ protects two disjoint working segments.

The paper is organized as follows. In the next section, we have a qualitative comparison of the BSSP, SSPO and SLSP protection schemes. In the following sections, we investigate efficient Integer Linear Programming (ILP) models in order to design minimum cost protection schemes, in Section IV for the BSSP scheme and in Section V for the SSPO scheme. Both models rely on column generation methods which have now been shown to be extremely efficient for solving highly combinatorial problems. Computational experiences are conducted in Section VI where it is shown that SSPO offers a very attractive solution, in terms of cost and bandwidth compromises. Conclusions are drawn in the last section.

\section{Shared Segment Protection}

\section{A. Generalities}

Although the BSSP segment protection scheme is less greedy (see Bouffard [6]) in terms of bandwidth than shared link protection, and with a nice compromise for recovery time between link and path protection, it lacks a full protection against node failures (i.e., failure of a device, as ADM, located at a node: in Figure 2(a) neither node $i_{1}$ nor $i_{2}$ are protected).

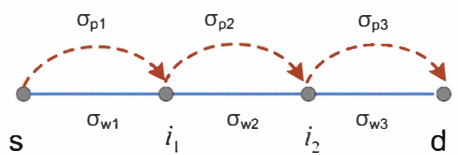

(a) BSSP with three optical hops

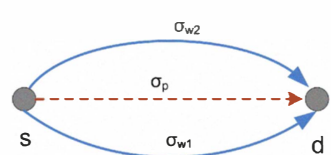

(b) Bandwidth sharing in BSSP
Fig. 2. BSSP protection scheme

Ho and Mouftah introduced in [5] the Short Leap Shared Protection (SLSP) scheme as an extension of BSSP, simultaneously protecting against node failure and fiber cut. Therein, the working path is subdivided into several equal length and overlapped segments, where each working segment is assigned (by the source node) a protection domain after the working path is selected. A drawback of the SLSP is the resulting increase of the number of optical hops and the higher complexity of the signaling operations. More recent investigations of SLSP can be found in, e.g., Tapolcai et al. [7].

In this paper, we consider a novel segment protection scheme, called Shared Segment Protection with Overlap (SSPO), which, as SLSP, offers a protection against both fiber cut and node failure, while taking advantage of the segmentation induced by the routing. The SSPO scheme consists in protecting each working segment simultaneously with at least another working segment (except for single hop requests) using overlapping protection segments instead of overlapping working segments as in SLSP. All different types of protection segments in the SSPO scheme, are shown on Figure 1. Note also that a protection segment can be shared with several working segments as long as they are pairwise disjoint.

While in the present study, we will not explicitly take into account shared risk group constraints, it is worth noting that they can easily be embedded in the proposed models by modeling two working segments belonging to the same risk group as conflicting working segments.

\section{B. BSSP vs. SSPO}

Depending on the network topology and the set of working segments (that depends in turn on the set of requests and on the GRWA algorithm used to define these segments), there is no dominance of either the BSSP or the SSPO protection scheme in terms of bandwidth and port cost as evaluated through the number of transport ports. Therefore, at equal or similar cost, SSPO should be favored over BSSP as it offers a better protection scheme, i.e., node and link failure vs. link failure only.

Let us examine two examples, with quite generic patterns, and which are depicted in Figures 3 and 6.

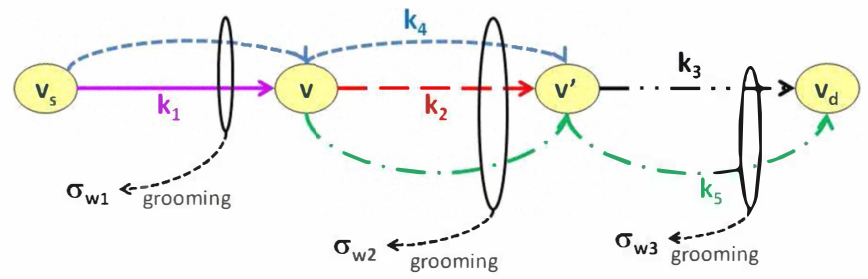

Fig. 3. Traffic Instance 1

The first traffic instance (Figure 3 ) is associated with a set of 5 requests, $\left\{k_{1}, k_{2}, k_{3}, k_{4}, k_{5}\right\}$ such that: $k_{1}: v_{s} \longmapsto v$ on 
1 segment, $k_{2}: v \longmapsto v^{\prime}$ on 2 segments, $k_{3}: v^{\prime} \longmapsto v_{d}$ on one segment, $k_{4}: v_{s} \longmapsto v^{\prime}$ on two segments, and $k_{5}: v \longmapsto v_{d}$ on one segment. $k_{1}$ and $k_{4}$ are groomed from $v_{s}$ to $v$ to form $\sigma_{w 1}$, $k_{2}, k_{4}$ and $k_{5}$ are groomed from $v$ to $v^{\prime}$ to form $\sigma_{w 2}, k_{3}$ and $k_{5}$ are groomed from $v^{\prime}$ to $v_{d}$ to form $\sigma_{w 3}$. A SSPO protection (Figure 5) requires 5 protection segments and 10 ports and is thus more expensive than a BSSP protection that uses only 3 protection segments and 6 ports as shown in Figure 4.

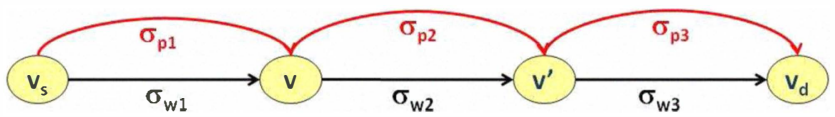

Fig. 4. BSSP: 3 Protection Segments and 6 Ports.

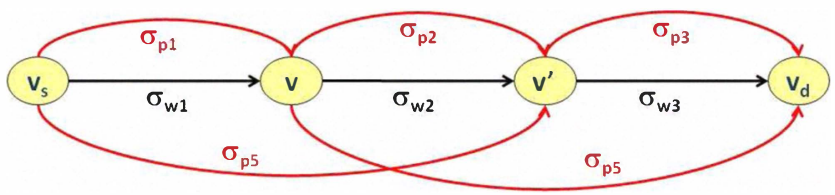

Fig. 5. SSPO: 5 Protection Segments and 10 Ports.

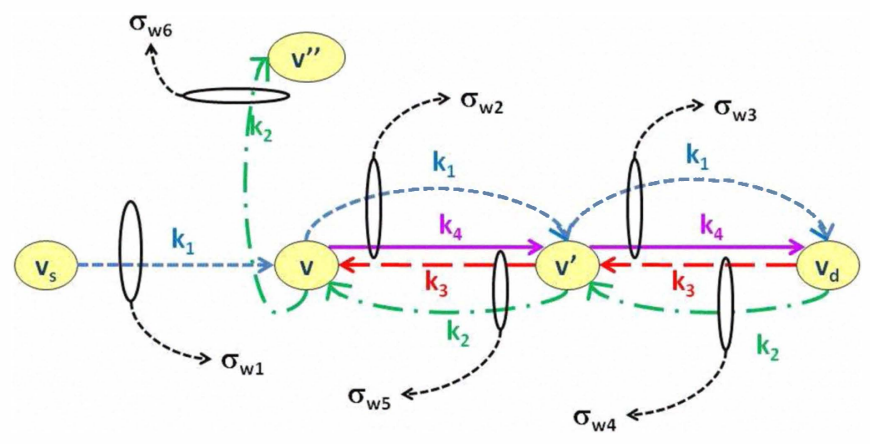

Fig. 6. Traffic Instance 2

However, there are traffic instances on which SSPO dominates BSSP. The second example, see Figure 6 , is associated with a set of 4 requests, $\left\{k_{1}, k_{2}, k_{3}, k_{4}\right\}$ such that $k_{1}: v_{s} \longmapsto$ $v_{d}, k_{2}: v_{d} \longmapsto v^{\prime \prime}, k_{3}: v_{d} \longmapsto v k_{4}: v \longmapsto v_{d}$. Let us assume that there are routed on wavelengths using the following working segments: $\sigma_{w_{1}}: v_{s} \longmapsto v, \sigma_{w_{2}}: v \longmapsto v^{\prime}$, $\sigma_{w_{3}}: v^{\prime} \longmapsto v_{d}, \sigma_{w_{4}}: v_{d} \longmapsto v^{\prime}, \sigma_{w_{5}}: v^{\prime} \longmapsto v$, $\sigma_{w_{6}}: v \longmapsto v^{\prime \prime}$ for request $k_{2}$. Only $k_{1}$ appears in $\sigma_{w_{1}}, k_{2}$ and $k_{3}$ are groomed together on $\sigma_{w_{2}}$ and on $\sigma_{w_{3}}, k_{1}$ and $k_{4}$ are groomed together on $\sigma_{w_{1}}$ and on $\sigma_{w_{4}}$, while $k_{6}$ appears in $\sigma_{w_{2}}$. For this example, SSPO protection is more economic than BSSP: It requires 8 ports (see Figure 8) vs. 12 ports for BSSP (see Figure 7).

Note also that depending again on the network topology and on the definition of the working segments, while it may not be possible for one of the protection scheme to define a protection for all requests (e.g. lack of available wavelengths), it may be possible for the other one, and vice-versa.

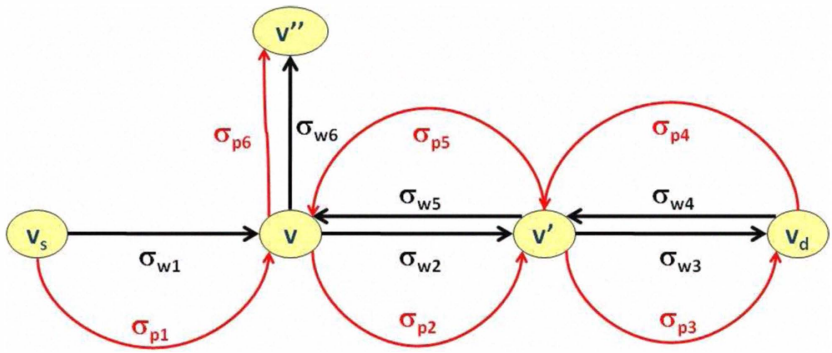

Fig. 7. BSSP: 6 Protection Segments and 12 Ports.

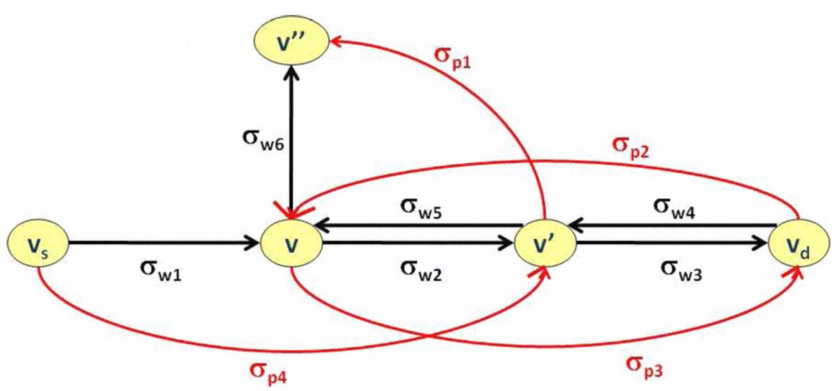

Fig. 8. SSPO: 4 protection segments and 8 Ports.

\section{NOTATION AND DEFINITIONS}

Consider a WDM network represented by a directed graph $G=(V, L)$ where $V=\left\{v_{1}, v_{2}, \ldots, v_{n}\right\}$ is the set of nodes in one to one correspondence with the set of network nodes, and $L=\left\{\ell_{1}, \ell_{2}, \ldots, \ell_{m}\right\}$ is the set of arcs, each arc being associated with a directional fiber link. Given $v \in V$, we denote by $\omega^{-}(v)$ and $\omega^{+}(v)$ the set of incoming and outgoing arcs of $v$ respectively.

The traffic is a set $K$ of requests such that, for each request $k \in K$, we are given its source $s_{k}$, its destination $d_{k}$, its bandwidth requirement and its working path represented by the set, $\mathcal{S}_{k}^{W}$, of working segments on which its lightpath has been groomed (we assume that the request bandwidth requirement is never greater than the transport capacities of the segments). Let $\mathcal{S}^{W}=\bigcup_{k \in K} \mathcal{S}_{k}^{W}$ be the set of all working segments. Note that each (unit) working segment $\sigma_{w}$ is characterized by a path $p\left(\sigma_{w}\right)$, from the source node of $\sigma_{w}$, denoted by $v_{s}\left(\sigma_{w}\right)$, to its destination node $v_{d}\left(\sigma_{w}\right)$ and a wavelength $\lambda\left(\sigma_{w}\right)$. It follows that each working segment $\sigma_{w}$ is associated with all requests groomed from $v_{s}\left(\sigma_{w}\right)$ to $v_{d}\left(\sigma_{w}\right)$ on wavelength $\lambda\left(\sigma_{w}\right)$ along path $p\left(\sigma_{w}\right)$.

Let $K^{i}$ be the set of requests with a working path using $i$ segments, $i=1,2,3$.

Denote by $\mathcal{S}_{w}^{P}$ the set of suitable protection segments for a working segment $\sigma_{w}$, in the context of the BSSP scheme, and by $\mathcal{S}_{w, k}^{P}$, the set of suitable protection segments for a working segment $\sigma_{w}$ and a request $k$ that is routed on it, in the context of the SSPO scheme. Let $\mathcal{S}^{P}=\bigcup_{\sigma_{w} \in \mathcal{S}^{W}} \mathcal{S}_{w}^{P}$ or $\mathcal{S}^{P}=\bigcup_{\sigma_{w} \in \mathcal{S}^{W}} \mathcal{S}_{w, k}^{P}$ be the set of protection segments/paths according to the selected protection schemes.

Two protection segments are in conflict if they use the same 
wavelength on a given fiber link. Two working segments can be protected by two conflicting protection segments if and only if they do not share any fiber link. For SSPO protection, we add the condition that they do not share any node except for their endpoints. Indeed, if a fiber link shared by two working segments is cut, we need to reroute each pair of working segments on two different alternative paths. We use the following parameters:

$$
\delta_{w w^{\prime}}= \begin{cases}1 & \text { if } \sigma_{w} \text { and } \sigma_{w}^{\prime} \text { can be protected by the same } \\ \text { protection segment } \\ 0 \quad \text { otherwise }\end{cases}
$$

for all pair $\left\{w, w^{\prime}\right\}$ of working segments. Note that $\delta_{w w^{\prime}}=$ $\delta_{w^{\prime} w}$.

\section{BSSP PROTECTION SCHEME}

In this section, we will restrict our attention to the BSSP protection. We propose to investigate a column generation formulation in order to find an optimal BSSP protection design. We will outline the main features and advantages of column generation formulations in the next paragraph, and then detail the proposed model in the following paragraphs. For the reader who is not familiar with column generation formulations and solutions, see, e.g., Chvatal [8] and Bamhart et al. [9].

\section{A. BSSP Protection Configurations}

Before setting the BSSP column generation model, denoted by CG-BSSP, we need to introduce the so-called wavelength $B S S P$ protection configurations. For each wavelength $\lambda$, we define a wavelength BSSP protection configuration, as a set of protection segments all routed on $\lambda$, which protect a given set of working segments that are not necessarily routed on wavelength $\lambda$. In order to reduce the number of configurations to be explored, we only consider the maximal ones, i.e., those that are not embedded in larger ones with identical cost.

A column generation model leads to a decomposition where the master problem takes care of selecting the best configurations, one for each wavelength, i.e., the set of configurations that minimizes the cost as evaluated by the number of transport ports. The pricing problem identifies, dynamically and one at a time, the most promising configurations, i.e., the configurations with a negative reduced cost (i.e., the objective of the pricing, see [8] if not familiar with linear programming tools). The pricing problem therefore handles the constraints associated with the definition of a protection segment, i.e., no link sharing between a working segment and its protection, protection sharing whenever it helps to reduce the cost. Note that due the efficiency of column generation, only a very small number of configurations will indeed need to be generated. Indeed, as soon as we cannot find any new configuration with a negative reduced cost, i.e., any new configuration that allows reduction of the master objective value, we can claim we have reach the optimal solution of the linear relaxation of the master problem. Then, one can use either rounding off techniques or
ILP solver to deduce an integer solution, see Bamhart et al. [9] for more details.

\section{B. CG-BSSP Master Problem}

Each variable $z_{c}$ of the master problem is associated with a configuration $c \in \mathcal{C}^{\lambda}$ for a given wavelength $\lambda: z_{c}=1$ if the $c$ configuration, $c \in \mathcal{C}^{\lambda}$, is selected on wavelength $\lambda$, and 0 otherwise. We denote by $B^{c}$ the unit cost of a configuration, $c \in \mathcal{C}$, which, in the present study, will be equal to the number of required ports (whether input or output ports), i.e.,

$$
B^{c}=\sum_{v \in V}\left(B_{v}^{c, \text { IN }}+B_{v}^{c, \text { OUT }}\right),
$$

where $B_{v}^{c, \text { IN }}$ and $B_{v}^{c, \text { OUT }}$ are the number of input and output ports at node $v$, respectively.

Let $\mathcal{C}^{\mathrm{BSSP}}$, or $\mathcal{C}$ for short when there is no confusion, be the overall set of potential wavelength BSSP protection configurations. Although it is a huge set following its definition, in practice only a small number of its elements (e.g., few hundred for large network and traffic instances) will need to be listed in order to get an optimal or a near optimal solution. Let $a^{C}$ be the column of the constraint matrix associated with the decision variable $z_{C}$. Each working segment is associated with a component of $a^{C}: a_{\sigma_{w}}^{C}=1$ if $\sigma_{w}$ can be protected under this configuration, and 0 otherwise. We then get the following mathematical model for the master problem:

$$
\min \sum_{\lambda \in \Lambda} \sum_{c \in C^{\lambda}} B^{c} z_{c}
$$

subject to:

$$
\begin{array}{ll}
\sum_{c \in C^{\lambda}} z_{c} \leq 1 & \lambda \in \Lambda \\
\sum_{\lambda \in \Lambda} \sum_{c \in C^{\lambda}} a_{\sigma_{w}}^{c} z_{c} \geq 1 & \sigma_{w} \in \mathcal{S}^{W} \\
z_{c} \in\{0,1\} & c \in C .
\end{array}
$$

Constraints (1) express that we must select at most one configuration per wavelength. Constraints (2) express the condition that each working segment must be protected at least once. Note that, in some cases, due to the search for maximal wavelength protection configurations, we may end up with "over" protection of some working segments: Not only it does not affect the port cost, but we could argue that in case of higher order failure, it might be useful.

\section{CG-BSSP Pricing Problems}

There are as many pricing problems as the number of wavelengths in order to take into account the wavelengths already assigned to the working segments. Consider the auxiliary graph $G_{\lambda}=\left(V, L_{\lambda}\right)$ where $L_{\lambda}=\{e \in L:(e, \lambda) \notin$ $\sigma_{w}$ for $\left.\sigma_{w} \in \mathcal{S}^{W}\right\}$. In order to define protection segments, we use a flow modeling formulation where each segment $\sigma_{p}$ that protects a given working segment $\sigma_{w}$ is associated with a unit flow from $v_{s}\left(\sigma_{w}\right)$ to $v_{d}\left(\sigma_{w}\right)$ where $v_{s}\left(\sigma_{w}\right)$ and $v_{d}\left(\sigma_{w}\right)$ denotes respectively the source and the destination nodes of the $\sigma_{w}$ segment. We therefore introduce flow variables $\varphi_{e \lambda}^{\sigma_{w}}$ 


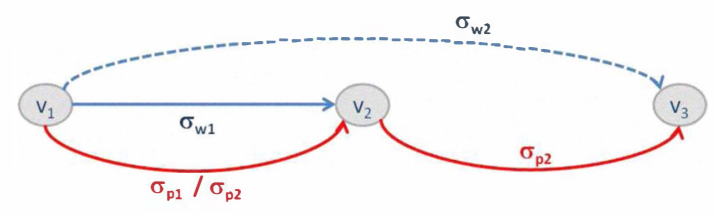

(a) Destination node $v_{2}=v_{d}\left(\sigma_{w 1}\right)$ requires an output port

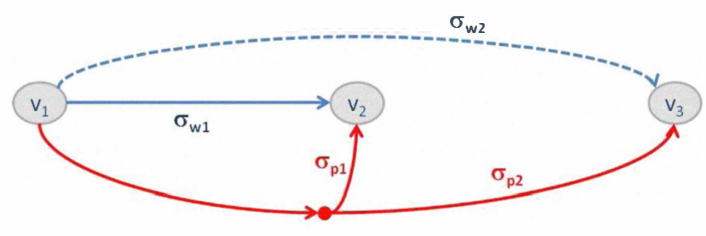

(c) Multi hop protection (split case)

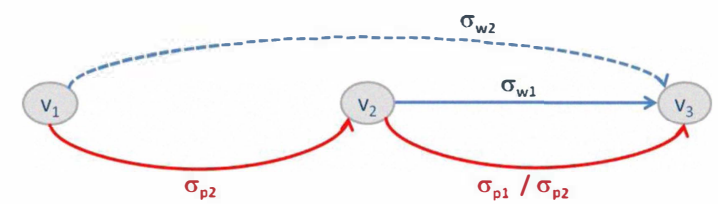

(b) Source node $v_{2}=v_{s}\left(\sigma_{w 1}\right)$ requires an input port

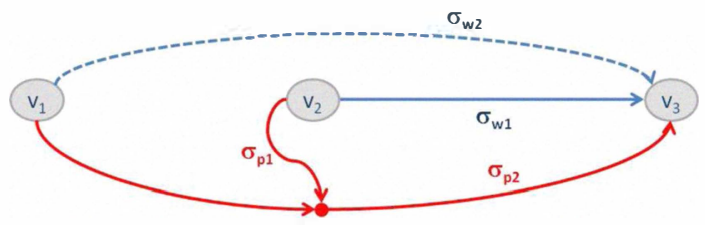

(d) Multi hop protection (merge case)

Fig. 9. Multi pop protection schemes assuming $\delta_{w 1, w 2}=1$

such that $\varphi_{e \lambda}^{\sigma_{w}}=1$ if $e$ supports a segment with wavelength $\lambda$ in order to protect $\sigma_{w}$, and 0 otherwise. Note that in case of a protection segment $\sigma_{p}$ shared by two link disjoint working segments $\sigma_{w}$ and $\sigma_{w}^{\prime}$ with the same endpoints $v_{s}$ and $v_{d}$, there will be an overall flow of value 2 (i.e., $\varphi_{e \lambda}^{\sigma_{w}}+\varphi_{e \lambda}^{\sigma_{w}^{\prime}}$ ) from $v_{s}$ to $v_{d}$ on all links $e$ of $\sigma_{p}$. Let us now study the mathematical formulation of the pricing problem for a given wavelength $\lambda$.

1) Objective Function: The objective function of a given pricing problem corresponds to the minimization of the reduced cost (see, e.g., [8] if not familiar with linear programming tools) that is defined by:

$$
\bar{B}^{c}=B^{c}+u_{0}^{\lambda}-u \cdot a^{c}=B^{c}+u_{0}^{\lambda}-\sum_{\sigma_{w} \in \mathcal{S}^{W}} u_{\sigma_{w}} a_{\sigma_{w}}^{c}
$$

for $c \in C^{\lambda}$, where $a_{\sigma_{w}}^{c}=\sum_{e \in \omega^{+}\left(v_{s}\left(\sigma_{w}\right)\right)} \varphi_{e}^{\sigma_{w}}$, and $u_{0}^{\lambda}$ and $u=\left(u_{\sigma_{w}}\right)_{\sigma_{w} \in \mathcal{S}^{W}}$ are the dual variables associated respectively with constraints $(1-\lambda)$ and $\left(2-\sigma_{w}\right)$ of the master problem.

As $B^{c}=\sum_{v \in V}\left(B_{v}^{c, \text { IN }}+B_{v}^{c, \text { ouT }}\right)$, we first need to compute the number of input $\left(B_{v}^{c, \text { IN }}\right)$ and output $\left(B_{v}^{c, \text { out }}\right)$ ports at node $v$. They can be evaluated as follows:

$$
\begin{array}{ll}
B_{v}^{c, \text { OUT }} \geq \sum_{e \in \omega^{+}(v)} \psi_{e}^{\text {oUT }} & v \in V \\
B_{v}^{c, \text { IN }} \geq \sum_{e \in \omega^{-}(v)} \psi_{e}^{\text {IN }} & v \in V, \\
\psi_{e}^{\mathrm{IN}}, \psi_{e}^{\text {OUT }} \in\{0,1\} & e \in L_{\lambda}
\end{array}
$$

where $\psi_{e}^{\mathrm{OUT}}$ and $\psi_{e}^{\mathrm{IN}}$ are decision variables, indicating whether or not an output/input port is required on link $e$.

In order to alleviate the notations, we now get ride of the $\lambda$ index until this end of this section and simply denote the flow variables by $\varphi_{e}^{\sigma_{w}}$. For the same reason, we will also omit the $c$ index.

Flow variables $\psi_{e}^{\text {OUT }}$ and $\psi_{e}^{\mathrm{IN}}$ must satisfy:

$$
\begin{array}{ll}
\psi_{e}^{\mathrm{IN}} \geq \varphi_{e}^{\sigma_{w}} & e \in \omega^{-}\left(v_{d}\left(\sigma_{w}\right)\right), \sigma_{w} \in \mathcal{S}^{W} \\
\psi_{e}^{\text {oUT }} \geq \varphi_{e}^{\sigma_{w}} & e \in \omega^{+}\left(v_{s}\left(\sigma_{w}\right)\right), \sigma_{w} \in \mathcal{S}^{W}
\end{array}
$$

$$
\begin{aligned}
& \alpha_{v}^{\text {oUT }} \geq \varphi_{e}^{\sigma_{w}} \quad v \in V: v=v_{d}\left(\sigma_{w}\right), \\
& e \in \omega^{-}(v), \sigma_{w} \in \mathcal{S}^{W} \\
& \psi_{e}^{\text {OUT }} \geq \varphi_{e}^{\sigma_{w}}+\alpha_{v}^{\text {oUT }}-1 \quad v \in V: v=v_{d}\left(\sigma_{w}\right) \text {, } \\
& e \in \omega^{+}(v), \sigma_{w} \in \mathcal{S}^{W} \\
& \alpha_{v}^{\mathrm{IN}} \geq \varphi_{e}^{\sigma_{w}} \\
& v \in V: v=v_{s}\left(\sigma_{w}\right) \text {, } \\
& e \in \omega^{+}(v), \sigma_{w} \in \mathcal{S}^{W} \\
& \psi_{e}^{\mathrm{IN}} \quad \geq \varphi_{e}^{\sigma_{w}}+\alpha_{v}^{\mathrm{IN}}-1 \quad v \in V: v=v_{s}\left(\sigma_{w}\right) \text {, } \\
& e \in \omega^{-}(v), \sigma_{w} \in \mathcal{S}^{W} \\
& \alpha_{v}^{\text {oUT }}, \alpha_{v}^{\text {IN }} \in\{0,1\} \quad v \in V
\end{aligned}
$$

Constraints (7) (resp. (8)) expresses that:

$$
\begin{array}{ll}
\psi_{e}^{\mathrm{IN}}=\max _{\sigma_{w} \in \mathcal{S}^{W}} \varphi_{e}^{\sigma_{w}} & e \in \omega^{-}\left(v_{d}\left(\sigma_{w}\right)\right), \sigma_{w} \in \mathcal{S}^{W} \\
\psi_{e}^{\mathrm{OUT}}=\max _{\sigma_{w} \in \mathcal{S}^{W}} \varphi_{e}^{\sigma_{w}} & e \in \omega^{+}\left(v_{d}\left(\sigma_{w}\right)\right), \sigma_{w} \in \mathcal{S}^{W} .
\end{array}
$$

In other words, $\psi_{e}^{\mathrm{IN}}=1$ (resp. $\psi_{e}^{\mathrm{ouT}}=1$ ) as soon as link $e \in$ $\omega^{-}\left(v_{d}\left(\sigma_{w}\right)\right)$ (resp. $\left.e \in \omega^{+}\left(v_{d}\left(\sigma_{w}\right)\right)\right)$ for $\sigma_{w} \in \mathcal{S}^{W}$ supports at least one protection flow.

Constraints (9) and (10) take care of the case illustrated in Figure 9(a) where a segment protection is reused as the first hop of another segment protection (in which case $\alpha_{v}^{\text {oUT }}=1$ for $v=v_{2}=v_{d}\left(\sigma_{w 1}\right)$ for the example of Figure 9(a)). Constraints (11) and (12) are similar constraints for the cases illustrated in Figure 9(b).

In order to detect the multi-hop protection schemes as illustrated in Figures 9(c) and 9(d), for all $v \in V$ such that $v$ is not the endpoint of a protected working segment in the current configuration, we need the following constraints:

$$
\begin{array}{ll}
\sum_{\sigma_{w} \in S} \max _{e \in \omega^{-}(v)} \varphi_{e}^{\sigma_{w}}-\sum_{\sigma_{w} \in S} \max _{e \in \omega^{+}(v)} \varphi_{e}^{\sigma_{w}} \leq|S| \alpha_{v} & S \subseteq \mathcal{S}^{W} \\
\sum_{\sigma_{w} \in S} \max _{e \in \omega^{+}(v)} \varphi_{e}^{\sigma_{w}}-\sum_{\sigma_{w} \in S} \max _{e \in \omega^{-}(v)} \varphi_{e}^{\sigma_{w}} \leq|S| \alpha_{v} \quad S \subseteq \mathcal{S}^{W} & \\
\psi_{e}^{\mathrm{IN}} \geq \varphi_{e}^{\sigma_{w}}+\alpha_{v}-1 & e \in \omega^{-}(v), \sigma_{w} \in S \\
\psi_{e}^{\text {OUT }} \geq \varphi_{e}^{\sigma_{w}}+\alpha_{v}-1 & e \in \omega^{+}(v), \sigma_{w} \in S \\
\alpha_{v} \in\{0,1\} & v \in V,
\end{array}
$$


where all working segments of $S$ are pairwise independent, i.e., $\delta_{w w^{\prime}}=1$ for any $w, w^{\prime} \in S$.

The above constraints are highly costly to include in the mathematical model, taking into account the very few multihop protection occurrences as illustrated in 9(c) and 9(d). We therefore decided not to include them but only check a posteriori their occurrences.

a) Constraints: Constraints of the pricing problem deal with the constraints associated with the definition of a proper wavelength protection configuration, they are as follows:

$$
\begin{aligned}
& \sum_{e \in \omega^{-}(v)} \varphi_{e}^{\sigma_{w}}=\sum_{e \in \omega^{+}(v)} \varphi_{e}^{\sigma_{w}} \quad \sigma_{w} \in \mathcal{S}^{W}, \\
& \sum_{e \in \omega^{+}\left(v_{s}\left(\sigma_{w}\right)\right)} \varphi_{e}^{\sigma_{w}}=\sum_{e \in \omega^{-}\left(v_{d}\left(\sigma_{w}\right)\right)} \varphi_{e}^{\sigma_{w}} \leq 1 \quad \sigma_{w} \in \mathcal{S}^{W} \\
& \sum_{e \in \omega^{-}\left(v_{s}\left(\sigma_{w}\right)\right)} \varphi_{e}^{\sigma_{w}}=\sum_{e \in \omega^{+}\left(v_{d}\left(\sigma_{w}\right)\right)} \varphi_{e}^{\sigma_{w}}=0 \quad \sigma_{w} \in \mathcal{S}^{W} \\
& \varphi_{e}^{\sigma_{w}}+\varphi_{e}^{\sigma_{w^{\prime}}} \leq 1+\delta_{w w^{\prime}} \quad e \in L_{\lambda} ; \sigma_{w}, \sigma_{w^{\prime}} \in \mathcal{S}^{W} \\
& \varphi_{e}^{\sigma_{w}} \in\{0,1\} \quad e \in L_{\lambda}, e \notin \sigma_{w}, \sigma_{w} \in \mathcal{S}^{W} \\
& \varphi_{e}^{\sigma_{w}}=0 \\
& e \in\left(L \backslash L_{\lambda}\right) \cup \sigma_{w}, \sigma_{w} \in \mathcal{S}^{W}
\end{aligned}
$$

Constraints (14) correspond to the flow conservation at intermediate nodes. Constraints (15) expresses that the flow starting at $v_{s\left(\sigma_{w}\right)}$ finishes at $v_{d\left(\sigma_{w}\right)}$ while (16) means that no flow arrives at $v_{s\left(\sigma_{w}\right)}$ and none leaves from $v_{d\left(\sigma_{w}\right)}$. Constraints (17) prevents two working segments in conflict (i.e., they share at least one fiber link) to be protected by the same protection segment. Equation (19) prevents a given link to be used both in a working segment and in its protection. Moreover, it forbids to use link $e$ with the $\lambda$ wavelength assignment in the definition of $\sigma_{p}$ if $(e, \lambda)$ is already included in a working segment.

\section{SSPO PROTECTION SCHEME}

In this section, we focus on the novel SSPO protection scheme with overlapping protection segments as described in Section II.

\section{A. The CG-SSPO Column Generation Model with Wavelength Configuration}

1) Master problem: The master problem has a similar mathematical expression than for the BSSP protection scheme, except the definition of the the wavelength protection configurations. In this case, wavelength SSPO protection configurations refers to the set of $(\lambda)$ protection segments that are shared by their protected working counterpart. The main difference between the BSSP and SSPO lies in the definition of the protection segments. For single hop working paths, they are the same as in BSSP: Their endpoints coincide with those of the working segment, while they cannot share any link of the working segments they protect. For 2-hop working paths, an SSPO protection is made of a single protection segment which has its two endpoints in common with those of the working path. For 3-hop working paths, an SSPO protection is either made of a single protection path (no difference with path protection) or of two protection segments that overlap over the second working segments, i.e., if the working path of request $k$ is made of three segments $\left(\sigma_{w}, \sigma_{w}^{\prime}, \sigma_{w}^{\prime \prime}\right)$, the first protection segment may start at $v_{s}\left(\sigma_{w}\right)$ and end at $v_{d}\left(\sigma_{w}^{\prime}\right)$, while the second protection segment would start at $v_{s}\left(\sigma_{w}^{\prime}\right)$ and end at $v_{d}\left(\sigma_{w}^{\prime \prime}\right)$. Notice that in both 2-hop and 3-hop working paths the working segment $\sigma_{w}^{\prime}$ is automatically protected if the others are. It is why we do not have to add specific constraints for them.

The SSPO protection, working segments of different requests can be either protected by overlapping segments in case of 3-hop working paths, or sequential non overlapping segments, see Figure 5. In this case, the protection segments and the working segments do not necessarily share the same endpoints. Constraints (20) are added in the master problem in order to protect different working segments, by overlapping or sequential segments, of different connections:

$$
\sum_{\lambda \in \Lambda} \sum_{C_{\lambda} \in \mathcal{C}} a_{\sigma_{w}, k}^{C_{\lambda}} z_{C_{\lambda}} \geq 1 \quad k \in K, \sigma_{w} \in \mathcal{S}^{k}
$$

2) Pricing Problem:

a) Objective: Minimize the reduced cost of the master:

$$
\bar{B}_{C_{\lambda}}=B_{C_{\lambda}}-\sum_{k \in K} \sum_{\sigma_{w} \in \mathcal{S}^{k}} u_{\sigma_{w}, k} a_{\sigma_{w}, k}^{C_{\lambda}}+u_{0}^{\lambda}
$$

where $u_{\sigma_{w}, k}$ is the dual variable associated with constraint $\left(20-\left(k, \sigma_{w}\right)\right)$.

For 1 hop:

$$
a_{\sigma_{w}, k}^{C_{\lambda}}=\sum_{e \in \omega^{+}\left(v_{s}\left(\sigma_{w}\right)\right)} \varphi_{e}^{\sigma_{w}, k}
$$

where $v_{s}\left(\sigma_{w}\right)=v_{s}^{k}$.

For 2 hops:

$$
\begin{array}{ll}
a_{\sigma_{w}, k}^{C_{\lambda}}= & \sum_{e \in \omega^{+}\left(v_{s}\left(\sigma_{w}\right)\right)} \varphi_{e}^{\sigma_{w}, k} \text { if } v_{s}\left(\sigma_{w}\right)=v_{s}^{k} \\
a_{\sigma_{w}, k}^{C_{\lambda}}=\sum_{e \in \omega^{-}\left(v_{d}\left(\sigma_{w}\right)\right)} \varphi_{e}^{\sigma_{w}, k} & \text { if } v_{d}\left(\sigma_{w}\right)=v_{d}^{k} .
\end{array}
$$

For 3 hops $\left(\sigma_{w}, \sigma_{w}^{\prime}, \sigma_{w}^{\prime \prime}\right)$ :

$$
\begin{array}{ll}
a_{\sigma_{w}, k}^{C_{\lambda}}=\sum_{e \in \omega^{+}\left(v_{s}\left(\sigma_{w}\right)\right)} \varphi_{e}^{\sigma_{w}, k} & \text { if } v_{s}\left(\sigma_{w}\right)=v_{s}^{k} \\
a_{\sigma_{w}^{\prime}, k}^{C_{\lambda}}=a_{\sigma_{w}^{\prime \prime}, k}^{C_{\lambda}} & \\
a_{\sigma_{w}, k}^{C_{\lambda}}=\sum_{e \in \omega^{-}\left(v_{d}\left(\sigma_{w}\right)\right)} \varphi_{e}^{\sigma_{w}, k} & \text { if } v_{d}\left(\sigma_{w}\right)=v_{d}^{k} .
\end{array}
$$

Let $\mathcal{S}^{W}=\mathcal{S}^{W_{1}} \cup \mathcal{S}^{W_{2}} \cup \mathcal{S}^{W_{3}}$, where $\mathcal{S}^{W_{i}}=\cup_{k \in K^{i}}\left(\mathcal{S}^{W} \cap\right.$ $\mathcal{S}_{k}^{W}$ ) is the set of working segments of requests with $i$ working segments (i.e., hops), $i=1,2,3$ and $\mathcal{S}_{k}^{W}$ is the set of working segments for request $k$. Denote by $v_{s}^{k}$ and $v_{d}^{k}$ respectively the source and the destination node of the working path of request $k$.

The number of output and input ports at node $v$ can be evaluated as in CG-BSSP with constraints (4), (5) and (6) 
using the flow variables $\psi_{e}^{\text {ouT }}$ and $\psi_{e}^{\mathrm{IN}}$. Those last variables must satisfy the same constraints as in the CG-BSSP model for single hop working paths. At intermediate nodes of protection segments, previous constraints (10)-(12) also apply. Single hop requests:

$$
\begin{array}{ll}
\psi_{e}^{\text {ouT }} \geq \varphi_{e}^{\sigma_{w}, k} & e \in \omega^{+}\left(v_{s}\left(\sigma_{w}\right)\right), k \in K_{1} \\
\psi_{e}^{\text {IN }} \geq \varphi_{e}^{\sigma_{w}, k} & e \in \omega^{-}\left(v_{d}\left(\sigma_{w}\right)\right), k \in K_{1}
\end{array}
$$

Two hop requests:

$$
\begin{array}{ll}
\psi_{e}^{\mathrm{OUT}} \geq \varphi_{e}^{\sigma_{w}, k} & e \in \omega^{+}\left(v_{s}\left(\sigma_{w}\right)\right), k \in K_{2} \\
\psi_{e}^{\mathrm{IN}} \geq \varphi_{e}^{\sigma_{w}^{\prime}, k} & e \in \omega^{-}\left(v_{d}\left(\sigma_{w}^{\prime}\right)\right), k \in K_{2}
\end{array}
$$

Three hop requests:

$$
\begin{array}{ll}
\psi_{e}^{\text {oUT }} \geq \varphi_{e}^{\sigma_{w}, k} & e \in \omega^{+}\left(v_{s}\left(\sigma_{w}\right)\right), k \in K_{3} \\
\psi_{e}^{\mathrm{IN}} \geq \varphi_{e}^{\sigma_{w}^{\prime \prime}, k} & e \in \omega^{-}\left(v_{d}\left(\sigma_{w}^{\prime \prime}\right)\right), k \in K_{3} \\
\psi_{e}^{\text {OUT }} \geq \varphi_{e}^{\sigma_{w}^{\prime}, k} & e \in \omega^{+}\left(v_{s}\left(\sigma_{w}^{\prime}\right)\right), k \in K_{3} \\
\psi_{e}^{\mathrm{IN}} \geq \varphi_{e}^{\sigma_{w}^{\prime}, k} & e \in \omega^{-}\left(v_{d}\left(\sigma_{w}^{\prime}\right)\right), k \in K_{3}
\end{array}
$$

b) Constraints: As in the previous model, the protection segment associated with a working segment will be defined by a unit flow following the SSPO protection scheme. We therefore need to specify the connection index together with the working segment to be protected as there might be different protection segments associated with a given working segment depending on the requests. Though the previous flow conservation constraints are also adapted and used in SSPO (they are not illustrated in SSPO because of space limitation).

$$
\sum_{e \in \omega^{+}\left(v_{s}\left(\sigma_{w}\right)\right)} \varphi_{e}^{\sigma_{w}, k}=\sum_{e \in \omega^{-}\left(v_{d}\left(\sigma_{w}\right)\right)} \varphi_{e}^{\sigma_{w}, k} \leq 1 \quad \sigma_{w} \in K^{1}
$$

Constraints (30) are used in single hop protection to set up the protection flow at the source and destination of protection segments.

$$
\begin{aligned}
\sum_{e \in \omega^{+}\left(v_{s}\left(\sigma_{w}\right)\right)} \varphi_{e}^{\sigma_{w}, k} & =\sum_{e \in \omega-\left(v_{d}\left(\sigma_{w^{\prime}}\right)\right)} \varphi_{e}^{\sigma_{w}, k} \leq 1 \\
\sum_{e \in \omega-\left(v_{d}\left(\sigma_{w}\right)\right)} \varphi_{e}^{\sigma_{w}, k} & =\sum_{e \in \omega^{+}\left(v_{s}\left(\sigma_{w^{\prime}}\right)\right)} \varphi_{e}^{\sigma_{w}, k}=0
\end{aligned}
$$

for all $v_{s}\left(\sigma_{w}\right)=v_{s}^{k},\left\{\sigma_{w}, \sigma_{w^{\prime}}\right\}=\mathcal{S}_{k}^{W}, k \in K^{2}$.

Constraints (31)-(32) are used to set up the protection flows and source and destination of 2-hop protection segments.

Similarly, for 3-hop working paths, constraints (33) express that the protection segment starts at $v_{s}\left(\sigma_{w}\right)$ and ends at either $v_{d}\left(\sigma_{w}^{\prime}\right)$ or $v_{d}\left(\sigma_{w}^{\prime \prime}\right)$, where $\sigma_{w}^{\prime}$ is the second working segment and $\sigma_{w}^{\prime \prime}$ the third.

$$
\begin{gathered}
\sum_{e \in \omega^{-}\left(v_{d}\left(\sigma_{w}^{\prime}\right)\right)} \varphi_{e}^{\sigma_{w}, k}+\sum_{e \in \omega^{-}\left(v_{d}\left(\sigma_{w}^{\prime \prime}\right)\right)} \varphi_{e}^{\sigma_{w}, k} \\
\geq \sum_{e \in \omega^{+}\left(v_{s}\left(\sigma_{w}\right)\right)} \varphi_{e}^{\sigma_{w}, k} \\
v_{s}\left(\sigma_{w}\right)=v_{s}^{k}, v_{d}\left(\sigma_{w}^{\prime \prime}\right)=v_{d}^{k},\left(\sigma_{w}, \sigma_{w}^{\prime}, \sigma_{w}^{\prime \prime}\right)=\mathcal{S}_{k}^{W}, k \in K^{3}
\end{gathered}
$$

Constraints (34) applies for the third working segment $\sigma_{w}^{\prime \prime}$, expressing that the protection segment starts at either $v_{s}\left(\sigma_{w}\right)$ or $v_{s}\left(\sigma_{w}^{\prime}\right)$ and ends at $v_{d}\left(\sigma_{w}^{\prime \prime}\right)$.

$$
\begin{gathered}
\sum_{e \in \omega^{+}\left(v_{s}\left(\sigma_{w}\right)\right)} \varphi_{e}^{\sigma_{w}, k}+\sum_{e \in \omega^{+}\left(v_{s}\left(\sigma_{w}^{\prime}\right)\right)} \varphi_{e}^{\sigma_{w}, k} \\
\geq \sum_{e \in \omega^{-}\left(v_{d}\left(\sigma_{w}^{\prime \prime}\right)\right)} \varphi_{e}^{\sigma_{w}, k} \\
v_{s}\left(\sigma_{w}\right)=v_{s}^{k}, v_{d}\left(\sigma_{w}^{\prime \prime}\right)=v_{d}^{k},\left(\sigma_{w}, \sigma_{w}^{\prime}, \sigma_{w}^{\prime \prime}\right)=\mathcal{S}_{k}^{W}, k \in K^{3}
\end{gathered}
$$

We do not need constraints for defining a protection for the second working segment of a 3-hop request, as its is protected by the same protection segment than the first or the last working segment.

\section{Computational RESUlts}

In this section we compare the performance of SSPO to BSSP. We consider in the performance evaluation two network topologies NSF [10] and COST239 [11] networks, and traffic distributions groomed following the approach in [6]. In the adopted traffic model, a working connection can span either (1, 2 or 3)-hop segments. The traffic load and distribution over the three type of connections are given in Table I under the header "Overall number of" and "Number of", respectively.

Both BSSP and SSPO optimization models are implemented and solved with the help of a (integer) linear programming solver (CPLEX package, [12]).

In NSF network (relatively sparse), the overlapping of protection segments depends on the distribution of the connections over the (1,2, and 3)-hop working segment classes. This factor (overlapping protection segments), which is illustrated in column 5 under the header "Overlapping protection segments", achieves its lower bound $75 \%$ when the number of 3-hop and 2-hop working segments are equal (see first row of Table I), and its highest bound $100 \%$ when the number of 2-hop connection is very low compared to 3-hop connections, see row 4. For all other cases where the number of 2-hop connections is higher than 3-hop, the number of overlapping protection segments is also high. In COST 239, more connected network compared to NSF, the number of overlapping protection segments is very high and clearly not highly dependent on the distribution of connections over the number of hops. It is in $[94 \%, 100 \%]$ of the total number of protection segments.

In column 6 and 7, we propose a comparison of the port cost between the two protection models. The cost of SSPO in terms of number of ports depend on the number of overlapping segments. In NSF, when compared to BSSP, which does not depend on the overlapping of segment, we see that they achieve comparable performance (comparable cost) when the number of 2-hop and 3-hop connections are comparable (first 3 lines), lower cost when 2-hop is lower (row 4), and almost higher cost whenever the number of 2-hop connection is high. In COST 239, this trend is not observed. The distribution of the traffic over the 2-hop and 3-hop connections does not 
TABLE I

COMPARISON OF BSSP AND SSPO PERFORMANCES

\begin{tabular}{|c|c|c|c|c|c|c|c|c|c|c|c|}
\hline & \multirow{2}{*}{$\begin{array}{r}\text { Overall } \\
\text { number of } \\
\text { requests }\end{array}$} & \multicolumn{3}{|c|}{ Number of } & \multirow{2}{*}{$\begin{array}{r}\text { Overlapping } \\
\text { segments } \\
(\%)\end{array}$} & \multicolumn{2}{|c|}{ Port cost } & \multicolumn{2}{|c|}{$\begin{array}{c}\text { Node } \\
\text { protection (\%) }\end{array}$} & \multicolumn{2}{|c|}{$\begin{array}{c}\text { Dual link } \\
\text { protection (\%) }\end{array}$} \\
\hline & & requests & requests & requests & & BSSP & SSPO & BSSP & SSPO & BSSP & SSPO \\
\hline \multirow[t]{10}{*}{$\overline{\mathrm{NSF}}$} & $\overline{32}$ & $\overline{12}$ & $\overline{12}$ & $\overline{8}$ & 7 & 38 & 42 & 53.3 & 100 & 36.4 & 65.2 \\
\hline & 89 & 33 & 46 & 10 & 90 & 63 & 66 & 0.0 & 100 & 0.0 & 42.6 \\
\hline & 94 & 41 & 47 & 6 & 83 & 64 & 60 & 0.0 & 100 & 0.0 & 38.6 \\
\hline & 100 & 89 & 8 & 3 & 100 & 66 & 58 & 22.2 & 100 & 9.5 & 15.2 \\
\hline & 166 & 48 & 85 & 33 & 88 & 84 & 75 & 5.6 & 100 & 6.7 & 49.1 \\
\hline & 181 & 48 & 85 & 48 & 94 & 58 & 73 & 17.7 & 100 & 21.1 & 54.9 \\
\hline & 241 & 70 & 102 & 69 & 89 & 94 & 78 & 12.7 & 100 & 14.8 & 53.2 \\
\hline & 324 & 114 & 161 & 49 & 90 & 72 & 79 & 38.9 & 100 & 31.5 & 56.7 \\
\hline & 354 & 114 & 183 & 57 & 84 & 58 & 76 & 4.8 & 100 & 6.4 & 46.8 \\
\hline & 546 & 206 & 262 & 78 & 99 & 60 & 78 & 42.3 & 100 & 33.6 & 57.0 \\
\hline \multirow[t]{6}{*}{ COST 239} & 47 & 13 & 17 & 17 & 94 & 56 & 42 & 13.6 & 100 & 9.4 & 55.7 \\
\hline & 99 & 27 & 50 & 22 & 98 & 44 & 48 & 6.9 & 100 & 6.5 & 50.5 \\
\hline & 106 & 35 & 54 & 17 & 100 & 45 & 45 & 6.4 & 100 & 13.3 & 47.0 \\
\hline & 170 & 91 & 54 & 25 & 100 & 60 & 56 & 9.6 & 100 & 7.1 & 40.3 \\
\hline & 174 & 48 & 81 & 45 & 100 & 53 & 68 & 26.0 & 100 & 23.4 & 57.0 \\
\hline & 204 & 59 & 94 & 51 & 100 & 37 & 50 & 24.3 & 100 & 22.2 & 55.9 \\
\hline
\end{tabular}

clearly affect the node cost in this case. This is mainly due to the high connectivity of COST 239 network. Though, richer connectivity in the protection capacity setup.

In terms of node protection, we observe that SSPO guarantees $100 \%$ protection against any single node failure even when the protection segment are not $100 \%$ overlapping. In this case, as the objective is to minimize the required protection capacity to provide $100 \%$ single segment protection, the protection segments tend to be paths. Though, also provide $100 \%$ protection against node failures. A very poor node protection is achieved by BSSP. This performance can be explained by the protection segments mainly made each of one fiber link.

Similarly for dual link failure protection, SSPO clearly outperforms BSSP, and usually offers more than $50 \%$ of duall link failure protection. The dual link failure protection in SSPO is at its lowest level when the number of 2-hop connections is far lower than the number of 3-hop connections (rows 4), its highest level when they are equal (row 1), and an in-between level in all other cases. Because of the rich connectivity, survivability to dual link failures is higher in the COST 239 network than the NSF in SSPO, but it is not sensitive to the distribution of $(2,3)$-hop connections.

\section{CONCLUSION}

In this paper we investigated a new shared segment protection scheme, SSPO, which ensures both node and link protection and compare it with the classical BSSP scheme. While both depends on the grooming results, i.e., the definition of the working segments, SSPO has a stronger dependency on the overlapping of the protection segments. Results show that with a small extra cost, SSPO offers an extended range of protection capabilities including node and dual-link failure protection. However, further investigations are needed in order to find out if the additional cost could be reduced thanks to a more efficient and adapted traffic grooming in order to reduce the number of overlapping segments.

\section{ACKNOWLEDGMENT}

Work of B. Jaumard has been supported by a Concordia Research Chair on the Optimization of Communication Networks and by a NSERC grant. Work of F. Huc and D. Coudert has been partially supported by ANR-JC OSERA, INRIA.

\section{REFERENCES}

[1] J. Ryan and R. Inc, "WDM: North American deployment trends," IEEE Communications Magazine, vol. 36, no. 2, pp. 40-44, 1998.

[2] B. Jaumard, C. Meyer, and B. Thiongane, "Comparison of ILP formulations for the RWA problem," Optical Switching and Networking, vol. 4, no. 3-4, pp. 157-172, November 2007.

[3] B. Chen, G. Rouskas, and R. Dutta, "On hierarchical traffic grooming in WDM networks," IEEE/ACM Transactions on Networking, vol. 16, no. 5, pp. 1226-1238, October 2008.

[4] B. Vignac, B. Jaumard, and F. Vanderbeck, "Hierarchical optimization procedure for traffic grooming in wdm optical networks," in Conference on Optical Network Design and Modeling - ONDM, February 2009, pp. $1-6$.

[5] P.-H. Ho and H. T. Mouftah, "A novel survivable routing algorithm for segment shared protection in mesh WDM networks with partial wavelength conversion," IEEE Journal of Selected Areas on Communications (JSAC), vol. 22, no. 8, pp. 1539-1548, 2004.

[6] A. Bouffard, "Dimensionnement GRWA et protection par segment dans les réseaux optiques WDM," Master's thesis, Université de Montréal, Canada, 2005.

[7] J. Tapolcai, P.-H. Ho, D. Verchère, T. Cinkler, and A. Haque, "A new shared segment protection method for survivable networks with guaranteed recovery time," IEEE Transactions on Reliability, vol. 57, pp. 272-282, June 2008.

[8] V. Chvatal, Linear Programming. Freeman, 1983.

[9] C. Barnhart, E. Johnson, G. Nemhauser, M. Savelsbergh, and P. Vance, "Branch-and-price: Column generation for solving huge integer programs," Operations Research, vol. 46, no. 3, pp. 316-329, May-June 1998.

[10] R. Hülsermann, S. Bodamer, M. Barry, A. Betker, C. Gauger, M. Jäger, M. Köhn, and J. Späth, "A set of typical transport network scenarios for network modelling," ITG Fachtagung Photonische Netze, Leipzig, Tech. Rep., 2004.

[11] P. Batchelor et al., "Ultra high-capacity optical transmission networks: Final report of action COST 239," Faculty of Electrical Engineering and Computing, University of Zagreb, Tech. Rep., 1999.

[12] ILOG CPLEX 11.0 Documentation, ILOG Inc., Gentilly, France, 2008. 